\title{
Jenseits des guten Geschmacks?
}

\section{Veganisierung im Alltag}

\author{
Rebecca Thrun
}

Journal für Psychologie, 28(2), 147-170

https://doi.org/10.30820/0942-2285-2020-2-147

CC BY-NC-ND 3.0 DE

www.journal-fuer-psychologie.de

\section{Zusammenfassung}

Der Beitrag fokussiert alltagsweltliche Moralisierungspraktiken im ethisch-moralisch begründeten Veganismus. Mittels der interpretativen Analyse ausgewählter Interviewsequenzen mit Veganer*innen aus Deutschland wird rekonstruiert, inwiefern vegane Überzeugungsarbeit eine gesellschaftskritische Perspektive auf die Normalisierung des Tierkonsums eröffnet. Aspekte der Nachhaltigkeit, Verantwortungsethik und Fürsorge werden von den Interviewpartner*innen angeführt, um die Notwendigkeit individueller und gesellschaftlicher Transformationsprozesse zu plausibilisieren. Gleichzeitig existieren andere (mitunter unbewusst wirkende) Beweggründe, die Mitmenschen an eigene vegane Sicht- und Lebensweisen anzugleichen. Handlungsmacht in der Gestaltung (intimer) sozialer Beziehungen steht dabei kontrastiv zur empfundenen Machtlosigkeit im Kontext (anonymer) gesellschaftlicher Strukturen und Prozesse des Tierkonsums. Eine Veganisierung des Umfeldes führt nicht selten zu Spannungen im Alltag. Die vegan lebenden Interviewpartner*innen versuchen diesen zu begegnen und greifen dabei präventiv auf erfolgversprechende Strategien veganer Überzeugungsarbeit zurück.

Schlüsselwörter: Veganismus, Verzicht, Affizierung, Verantwortung, Speziesismus, Mitgefühl, qualitative Forschung

\section{Summary}

Beyond good taste? Veganization in everyday life

The contribution focuses on everyday moralizing practices in ethically-morally based veganism. By means of the interpretative analysis of selected interview sequences with Vegans from Germany, I reconstruct in how far vegan work of conviction opens up a society-critical perspective on the normalization of animal consumption. The interviewees relate to aspects of sustainability, ethics of responsibility and care in order to plausibilise the necessity of individual and social transformation processes. At the same time, other (sometimes unconscious) motives play a role. They aim at persuading fellow human beings to share the same convic- 
tions and pursue a vegan lifestyle. The power to act in shaping (intimate) social relationships contrasts with the felt powerlessness in the context of (anonymous) social structures and processes of animal consumption. A veganisation of the social environment often leads to tension in everyday life. The interviewees seek to address these tensions preemptively by making use of promising strategies of vegan persuasion.

Keywords: Veganism, renunciation, affection, responsibility, speciesism, compassion, qualitative research

\section{Theoretische Perspektivierung: Mehrdeutigkeiten im Veganismus}

Vegane Denk- und Handlungsmuster finden seit einigen Jahren starke Verbreitung in westlichen Industrieländern und sind von zunehmendem Interesse für Wissenschaft und Forschung. Untersuchungen eines gesundheitlich motivierten Ernährungsstils (Leitzmann und Keller 2013), lebensstilistischer Ausprägungen (Grube 2009) einer veganen Community - unter anderem mit szenetypischen und jugendkulturellen Aspekten (Hitzler und Niederbacher 2010; Rinas 2012; Schwarz 2005) - oder auch der Fokus auf vegane Formen von Tierrechts-Aktivismus mit politischen Ansprüchen (Rude 2013) verdeutlichen, dass es sich beim Veganismus um ein polyvalentes Phänomen (Boesch 2000, 2006) handelt, an das unterschiedliche Motivationslagen und Praktiken anknüpfen. Angela Grubes (2009) Differenzierung in ethisch-moralische, gesundheitliche, ökologische, ökonomische und religiös-spirituelle Ausprägungen im Veganismus, die einen Verzicht auf tierische Produkte begründen, sind ein erster Zugang zum Forschungsgegenstand. Die Grenzen sind jedoch mitunter fließend oder lösen sich unter Begrifflichkeiten der (Mit-)Verantwortung oder (Für-)Sorge (Thrun 2017) teilweise auf. Im Zuge der Etablierung einer veganen Alltagskultur schärft sich vor allem der Fokus auf eine Verortung jenseits der Räume privaten Geschmacks und Genusses: Hier geht es um die Frage, inwiefern vegane Überzeugungen und Praxen von Anhänger*innen nicht bloß als optional, sondern als obligatorisch für eine zukünftige Gesellschaft gerahmt werden und welche sozialen Konflikte daraus resultieren. Vegane Sinnstrukturen stehen in einer hegemonial karnivoren Gesellschaft (Joy 2013, 2017) nämlich in einem Spannungsverhältnis zu Deutungsmustern, die das Wohl der Tiere entweder nicht einbeziehen oder anders definieren, beispielsweise zwischen Haus- und Nutztieren unterscheiden. Darüber hinaus existieren Schnittmengen zwischen veganen Selbstbildern und einem ökologischen Selbst (Straub und Ruppel 2017), die einen Fokus auf globale und zukünftige Auswirkungen lokaler Handlungspraxen setzen und die bestehende Bedrohung der Umwelt sowie die Lebensbedingungen zukünftiger Generationen mit einbeziehen. In 
diesem Zusammenhang wird ein zunehmendes Bewusstsein für die Krisenhaftigkeit etablierter Gewohnheiten des Tierkonsums ausgebildet, welcher hier und nachfolgend als Kurzform eines Ge- und Verbrauchs von Produkten tierischen Ursprungs verwendet wird. Ansprüche einer Verbreitung veganer Überzeugungen und Praxen im Sinne einer Veganisierung der Gesellschaft sind dabei nicht ausschließlich im Kontext (neuer) sozialer Bewegungsformen (Rucht 1994) angesiedelt. Auch auf alltagsweltlicher Ebene zeichnen sich Praktiken der Moralisierung ab, die an explizite Transformationsansprüche geknüpft sind. Für den in diesem Beitrag verfolgten Ansatz ist es bedeutsam, diese Formen der Moralisierung im Spannungsfeld einer dominant etablierten Normalisierung des Tierkonsums sowie seiner sich partiell etablierenden (mitunter radikalen) Ablehnung zu rekonstruieren und in ihrer psychosozialen Funktionsweise zu begreifen. Dabei geht es um die Frage, wie Unterlassungen (Straub 1999) des Konsums tierischer Produkte (als grundlegende vegane Praktiken) nicht nur von einem selbst, sondern auch (explizit oder implizit) von dem eigenen Umfeld gefordert werden. Wie werden diese Forderungen konkret ausgestaltet und welche Auswirkungen hat dies auf die Regulation sozialer Beziehungen? Im nächsten Abschnitt wird Moral(-isierung) zunächst aus kulturpsychologischer Perspektive beleuchtet, um die Komplexität moralischer Gedanken und Empfindungen sowie ihre enge Verwobenheit mit der (Um-)Deutung von Normalität und Abweichung aufzuzeigen. Anschließend wird mit Bezug auf Melanie Joy (2017) und Ernst-Dieter Lantermann (2016) verdeutlicht, inwiefern Untersuchungen zum Veganismus selbst bereits durch Normalitätspostulate geprägt sind und dies wesentlich auf das Verständnis moralischer Implikationen einwirkt. Vorstellungen der (Ir-)Rationalität sind dabei nicht unabhängig von gesellschaftlichen Machtverhältnissen zu denken. Im dritten Abschnitt werden ausgewählte Selbsterzählungen von Veganer*innen einer Analyse unterzogen, um empirisch aufzuzeigen, wie Moralisierungspraktiken im Veganismus gestaltet sind und plausibilisiert werden. Dabei wird herausgearbeitet, dass der eigene Tierkonsum in der Vergangenheit als Kontrastfolie dient, um eine Transformation des Selbst- und Weltverständnisses (Straub 2010) zu artikulieren. Die reflexive Auseinandersetzung mit vergangenen Selbstverständlichkeiten (eigenen Tierkonsums) ermöglicht es, Potenziale und Grenzen eigener Moralisierungspraktiken auszuloten.

\subsection{Die komplexe Verknüpfung moralischer Gedanken und Empfindungen}

Moral wird in diesem Beitrag zunächst als konstitutiv für soziale Beziehungen verstanden. Sie steht in einem intrinsischen Zusammenhang mit Handlungsmustern (Straub und Ruppel 2017), denn »moralische Werte und damit verwobene soziale Normen konstituieren oder regulieren unser Tun und Lassen « (ebd., 16 mit Bezug auf Straub 1999, 127). Welche konkreten Ausprägungen moralische Überzeugungen annehmen, 
wer oder was in ihre sowohl konkreten Artikulationen, aber auch ihre impliziten und unbewussten Wirkungsweisen einbezogen, für nicht relevant erachtet oder ausgeschlossen wird, ist von gesellschaftlichen Strukturen und Prozessen mit beeinflusst und dabei potenziell variabel. Insbesondere diese Grenzziehungen der Bezugspunkte für moralische Empfindungen und Gedanken sind wesentlicher Fokus tier- und verantwortungsethischer Überlegungen. In der Tierethik werden moralische Positionen wie Kants kategorischer Imperativ, der Utilitarismus oder eine Ethik der Fürsorge (siehe dazu Wolf 2008) - bezüglich ihres Integrationspotenzials geprüft, um eine allgemeine Ethik zu entwickeln, die auch die Tiere mit einbezieht, »da sich an der Frage der Tiere besonders gut zeigt, wo die Grenzen einer Position liegen, welche Überzeugungen sie zu integrieren vermag und welche sie zu sprengen drohen « (ebd., 10). Eine Reflexion dieser metaphysischen Positionen, um daraus Gebote und Verbote abzuleiten, greift hingegen für ein Verständnis veganer Moralisierungspraktiken zu kurz. Sowohl moralische Subjekte als auch Handlungssituationen, die ein moralisches Urteil einfordern, sind in ein Geflecht aus gesellschaftlichen Konventionen, biografischen Relevanzen und persönliche Gefühlslagen eingebunden: »Tatsächlich stellen sich Menschen moralische Fragen selten in Reinkultur, sondern fast immer eingebettet in andere - existenzielle, ökonomische, politische et cetera Fragen, die zumeist eine starke kulturelle Färbung aufweisen « (Herzog 2018, 296). Die komplexe Vernetzung der (Rekonstruktion von) Vergangenheit, Gegenwart und (Antizipation der) Zukunft eines Subjekts (Boesch 2006) spielt in diesem Zusammenhang eine wesentliche Rolle, um nicht nur eine Verfestigung von moralischen Überzeugungen, sondern auch eine (mögliche) Veränderung in den Blick zu nehmen und sowohl Handlungspotenziale als auch Grenzen zu verstehen. Durch diese theoretische Perspektivierung können Grenzen kognitiver Urteilsbildung (Kohlberg 1996) angemessen verstanden werden, da die Handlungspraxis eine (moralische) Überzeugung bereits mit konstituiert und dabei nicht losgelöst von gesellschaftlichen Strukturen und Prozessen ist.

\subsection{Vorstellungen von Normalität und Abweichung: Die Relationalität von Tierkonsum und Veganismus}

Moralische Werte können unterschiedliche Nähe zu Normen haben (Joas 1999) und auch in einem konfligierenden Verhältnis zu diesen stehen. Dies kann so weit führen, dass Illegalität für einen progressiv und emanzipatorischen Zweck umgedeutet sowie legitimiert wird, wie beispielsweise im Kontext von Tierbefreiungsaktionen zu beobachten ist (Friedrichs 1997; Rude 2013). Moralität ist dabei relational zu Vorstellungen von Gewalt(-losigkeit): »Beides - Gewalt und Moral - steht für die Sozialität des Menschen - das eine im Negativen, das andere im Positiven. Insofern hat es die Moral 
wesentlich mit der Regulierung der Art und Weise, wie Menschen miteinander umgehen, zu tun « (Herzog 2018, 297). Hier inhärent: Moralisches Handeln wird nicht nur exklusiv dem Menschen zugeschrieben, sondern bezieht sich auch auf diesen. Im Kontext von Moralität sowie Moralisierungspraktiken im Veganismus sind in diesem Zusammenhang zwei (miteinander verschränkte) Ebenen von Bedeutung: erstens eine Kritik an - als gewaltförmig wahrgenommenen - Strukturen und Prozessen in der Nutztierhaltung und damit verwobene gängige Praxen des Tierkonsums und zweitens explizite und implizite Einflüsse dieser Kritik auf der Ebene sozialer Beziehungen - mit der Gefahr, dabei möglicherweise nicht nur Spuren, sondern auch Narben beim Anderen zu hinterlassen. Interessant ist, dass weltanschauliche Veganer*innen (Leitzmann und Keller 2013) als Moralist*innen adressiert werden. Dies wird auch durch die Perspektive der Fragestellung transparent, die im Rahmen der Forschung hinsichtlich des Phänomens eingenommen wird: Warum konsumieren Menschen keine Tiere? ist zu unterscheiden von Warum konsumieren Menschen Tiere? In diesem Kontext zeichnen sich bereits spezifische Vorstellungen von Normalität und Abweichung ab. Hier schließt Joy (2017) an, die eine Adressierung von Veganer*innen als Moralist*innen mit Rekurs auf die unsichtbaren Mechanismen eines karnistischen Systems begründet:

»Carnism is the opposite of veganism. We tend to think that only vegans (and vegetarians) bring their beliefs to the dinner table. But when eating animals is not necessity, which is the case for many people in the world today, it is a choice - and choices always stem from beliefs $\ll($ ebd., 76).

Veganer*innen würden bereits durch ihre bloße Anwesenheit in einer moralisierenden Appellfunktion wahrgenommen, ohne diese tatsächlich auch zu intendieren, da sie hinsichtlich gängiger Praxen des Tierkonsums abweichen und dies begründen müssen. Unsichtbare, institutionalisierte und erlernte Mechanismen eines Systems, das bestimmten Lebewesen (Haustiere) Empfindungs- und Leidensfähigkeit zuschreibt und das Leid der anderen (Nutztiere) verschleiert, markieren den Tierkonsum dabei als normal, notwendig und natürlich und setzen seine Ablehnung als legitimierungsbedürftig voraus: »Carnism creates a paradoxical mentality. We would feel guilty eating certain animals, yet we take pleasure in consuming others « (ebd., 77). Diese systematische Logik eines ambivalenten Verhältnisses zwischen Mensch und Tier sei institutionell verfestigt (sozialer Karnismus) und durch Lernprozesse wie Rationalisierungsstrategien, Verdrängungs- und Abwehrmechanismen (psychologischer Karnismus) vom Individuum internalisiert und inkorporiert:

»[B] ecause we naturally feel empathy towards animals and don't want them to suffer, carnism must provide us with a set of tools to override our conscience, our natural resistance, 
so that we support a system that we would otherwise likely find deeply offensive « (ebd., 78).

Der Veganismus als opponierendes System, verkörpert durch die anwesenden Veganer*innen, stelle Selbstverständlichkeiten des Tierkonsums zwar infrage, allerdings würden Prozesse der negativen Stereotypisierung und Vorurteilsbildung gegenüber vegan lebenden Menschen als Schutz dienen, diese Irritationen auch in neue Denkund Handlungsgewohnheiten zu übersetzen. Joy spricht in ihren Überlegungen einen wesentlichen Punkt an: Wahlmöglichkeiten, bestimmte Ernährungs- und Lebensstile auszubilden, machen ideologische Wirkungsmechanismen transparent. Grundlage ist die Optionenvielfalt pluralistischer Gesellschaftsformationen, die sowohl Möglichkeitsräume eröffnen, als auch ein Gefühl der Bedrohung (des eigenen Selbst) generieren können: »Anderes und Fremdes, zumal wenn es mit irgendeiner Art von impliziter Anforderung oder expliziter Aufforderung einhergeht, sich selbst zu ändern, kann die Betroffenen ängstigen und überfordern«(Straub 2016, 148). Negative Affekte wie Angst, Aversion oder (moralischer) Ekel dienen dazu, diese Bedrohungen abzuwehren, verweigern gleichzeitig den offenen Dialog und bedingen eine dichotomisierende Wahrnehmung von Freund und Feind. Indem Joy insbesondere die (unbewusste) Wahrnehmung von Veganer*innen als mögliche Bedrohung für Nicht-Veganer*innen fokussiert, verstärkt sie diese Dichotomisierung, ohne den Fokus zusätzlich erstens auf die Wechselseitigkeit und Verwobenheit von Prozessen der Stigmatisierung und Gegenstigmatisierung zu legen, und zweitens interne Konfliktlinien zwischen vegan lebenden Menschen einerseits und eigenen inneren Spannungen andererseits zu berücksichtigen. Veganer*innen positionieren sich beispielsweise auf bestimmte Weise zu Aspekten der Missionierung, des Dogmatismus und der Militanz, die dem Feld inhärent sind. Sie werden zum einen im Kontext negativer Stereotypisierung, medialer Inszenierung des Veganismus oder im politischen veganen Tierrechts-Aktivismus erzeugt, sind zum anderen aber auch explizit an spezifische vegane Selbst- und Weltverständnisse geknüpft.

\subsection{Veganisierungen als fanatische Praxis?}

Tendenzen radikaler und fanatischer Strömungen im Veganismus hat sich Lantermann (2016) gewidmet. Dabei legt er ein stufentheoretisches Verständnis einer Differenz zwischen Radikalität und Fanatismus zugrunde:

»Im Unterschied zu den radikalen Veganern versteht ein fanatischer Veganer seine eigene vegane Lebensphilosophie nicht nur für sich, sondern für alle Menschen als letzten, abso- 
luten moralisch-ethischen Maßstab (die Gewährleistung von Tierwohl als absolutes und nicht verhandelbares Prinzip). Nicht vegan lebende Menschen gilt es zu missionieren oder, wenn die Widerstände zu groß werden, zu bekämpfen « (ebd., 157).

Die wichtigste Differenz sei dabei die Intensität der Gefühle und die Enthemmung als emotionaler Impuls gegenüber vernunftorientierten Abwägungen. Hass gegenüber der »anderen « Gruppe (der Nicht-Veganer*innen) führe zu einer Immunisierung bezüglich Kritik:

»Während der Radikalisierte zumindest noch in Grenzen zur Reflexion, Selbstkritik, zu Dialog und Kompromissen bereit und fähig ist, wäre dies für den Fanatiker ein unverzeihlicher Verrat an seiner heiligen gerechten Sache, die es in aller Entschiedenheit, Kompromisslosigkeit und Reinheit in die Tat umzusetzen gilt « (ebd., 166).

Die Möglichkeit der Herausbildung unterschiedlicher Lebens- und Ernährungsstile in einer pluralistischen Gesellschaft wird in diesem Zusammenhang als Verunsicherung und Gefahr der Entfremdung erlebt, welche durch Tendenzen der Selbstverschließung und Vereindeutigung präveniert werden. Ein daraus resultierendes moralisch überlegenes Auftreten und eine daran gekoppelte Entwertung des Anderen generiere Reaktanz bei nicht vegan lebenden Menschen, die eine Begrenzung ihrer Möglichkeitsräume empfinden und die Frontenbildung umgekehrt noch verstärken würden: »Fassen die Betroffenen die Nötigung als Versuch auf, ihre Freiheit einzuschränken, antworten sie mit einer Aufwertung ihrer bedrohten Handlungsalternative: Sie räumen ihr schlechtes Gewissen und ihre früheren Skrupel wegen ihres Fleischkonsums beiseite « (ebd., 161). Joys Überlegungen zum Karnismus verweisen darauf, dass speziell auch Prozesse negativer Stereotypenbildung gegenüber vegan lebenden Menschen an einer Frontenbildung beteiligt sind und die Anwesenheit beziehungsweise Abwesenheit von Anerkennung und gegenseitigem Verständnis wesentlich auf vegane Selbstpräsentationen und Moralisierungspraktiken einwirken. Nicht nur der Veganismus produziert als ideologisches Überzeugungssystem Ein- und Ausschlussmechanismen, auch ein System der Normalisierung des Tierkonsums ist (mitunter weit intransparenter) daran beteiligt. In diesem Beitrag soll gezeigt werden, dass Moralvorstellungen mit Universalisierungsansprüchen im Veganismus differenzierter zu betrachten sind, als sie voreilig unter Maßstäben der Radikalität oder des Fanatismus zu subsumieren. In diesem Zusammenhang ist die Möglichkeit sozialen Wandelns immer auch an eine (radikale) Kritik an gesellschaftlichen Strukturen und Prozessen geknüpft. Darüber hinaus bleibt in Lantermanns Überlegungen weitgehend unbeachtet, dass ein veganer Transformationsanspruch selbst bereits mehrdeutig ist (beispielweise als Neubestimmung eines Mensch-Tier-Verhältnisses im Sinne einer friedlichen Ko-Existenz von 
Mensch und Tier; als Abwehr einer erwarteten Klima-Katastrophe; als Gewährleistung von globaler Verteilungsgerechtigkeit etc.) und lediglich die Praxis des Verzichts auf Tierkonsum auf einen kleinsten gemeinsamen Nenner verweist. Insgesamt dienen die Perspektivierungen von Joy und Lantermann dazu, den Veganismus sowohl in einer Relationalität zur Normalisierung des Tierkonsums zu begreifen, als auch interne Differenzlinien zu berücksichtigen, die einer Dichotomisierung von vegan und nicht-vegan entgegenwirken und die komplexen Verflechtungen veganer Moralisierungsbestrebungen anerkennen. Die folgende Analyse ausgewählter Auszüge aus Interviewgesprächen mit Veganer*innen soll exemplarisch rekonstruieren, wie vegane Überzeugungsarbeit als - Lernprozessen entwachsene - Kommunikationsstrategie verstanden werden kann und dabei affektive Färbungen aufweist, die situativ mit einwirken. Ein Wechselspiel aus sowohl nach innen als auch nach außen gerichteter Regulation ist dabei ebenso zu beobachten wie ein strategisches Verständnis von Affizierungen des Gegenübers, um eine Veganisierung (als Bestreben, die Ablehnung des Konsums tierischer Produkte zu verbreiten) auch jenseits der Grenzen des rationalen Arguments zu ermöglichen. Fremd- und Selbstregulierung können aus kulturpsychologischer Perspektive als verwoben verstanden werden, da eine intrinsische Beziehung zwischen Kultur und Psyche (vgl. Straub und Chakkarath 2019) besteht und »psychische Phänomene grundsätzlich als kulturell konstituiert, reguliert oder moduliert gelten « (ebd., 25). Nachfolgend wird rekonstruiert, dass diese Regulierung des Selbst und der Mitmenschen bei den Interviewpartner*innen damit einhergeht, sowohl eigene als auch fremde Gedanken, Empfindungen und Gewohnheiten mitunter als willentlich steuerbar zu deuten. Dies erzeugt bestimmte Vorstellungen eigener Handlungsmacht und ihrer Grenzen.

\section{Empirische Analysen: Macht und Möglichkeiten des veganen Selbst in einer defizitären Welt}

Das empirische Datenmaterial, welches die Grundlage für diesen Beitrag bildet, wurde aus insgesamt zwölf Interviews ausgewählt, die zum einen aus Vorstudien im Kontext einer unveröffentlichten Masterarbeit stammen (Thrun 2015) und zum anderen im Rahmen eines laufenden Promotionsprojektes im Jahr 2019 erhoben wurden. Wesentlich für die Stichprobe ist, dass vegane Überzeugungen von den Akteur*innen nicht als optional, sondern als obligatorisch begriffen werden und die konkreten Voraussetzungen und Ausprägungen dieser angenommenen Zukunfts- sowie Mehrheitsfähigkeit des Veganismus - in Anlehnung an ein theoretisches Sampling (Glaser und Strauss 1998) - erst im Verlauf des (noch andauernden) Forschungsprozesses ausdifferenziert werden. Die Interviewpartner*innen wurden über explizit vegane 
Veranstaltungen und E-Mail-Verteiler rekrutiert. Die Interviews haben eine Dauer zwischen knapp einer und knapp drei Stunden. Neben Einzelinterviews wurden auch zwei Paarinterviews (Wimbauer und Motakef 2017) sowie ein Familiengespräch durchgeführt. In Anlehnung an die relationale Hermeneutik (Straub 2010) wurden die veganen Selbsterzählungen und die paar- sowie gruppendynamischen Interviewsequenzen komparativ analysiert (Glaser und Strauss 1967; Strauss 1991; Straub 2010), um im Sinne einer hermeneutischen Problematik des Sinnverstehens (vgl. Straub 2010, 46 nach Rabinow und Sullivan 1979) und ausgerichtet an Maßstäben der Transparenz und intersubjektiven Nachvollziehbarkeit die Komplexität des Veganismus als Sinn- und Bedeutungskonstrukt zu rekonstruieren. Dies spiegelt sich auch in der Gestaltung der Interviewsituation wider: Zur Generierung umfassender (biografisch eingebetteter) Narrationen (in Anlehnung an Schütze 1983) wurden die Interviews mit einer erzählgenerierenden Frage eröffnet. Im späteren Verlauf wurden flexibel anwendbare problemzentrierte Leitfadenfragen gestellt. Letztere werden in Anlehnung an Witzel (1989) zur Rekonstruktion subjektiver Relevanzsysteme herangezogen, um sowohl gegenstandsorientiert als auch prozessorientiert zu verfahren. Exemplarisch wurden Auszüge aus zwei Interviews ausgewählt, in welchen Moralisierungsbestrebungen in der alltagsweltlichen Kommunikation im familiären und partnerschaftlichen Umfeld eine wesentliche Rolle spielen. Das erste Interview wurde mit einem veganen Buddhisten (Ben) ${ }^{1}$ geführt, der sich für ein Verbot von Tierversuchen engagiert. Dabei stellt er retrospektiv sein selbstzugeschriebenes militantes Verhalten in der Vergangenheit als Störfaktor seiner sozialen Beziehungen heraus. Er begründet damit seinen selbst als moderat eingeschätzten gegenwärtigen Umgang mit nicht-veganen Lebenspraxen, versteht sich aber auch weiterhin als Impulsgeber mit Transformationsansprüchen. Das zweite Interview wurde mit einem veganen Paar (Maja und Flo) geführt, welches den Raum der Kunst und sogenannten Artivismus (veränderungsorientiertes Handeln mit künstlerischem Anspruch) als wesentliches Kommunikationsmittel benennt, um vegane Botschaften über einen Akt des Zeigens zu vermitteln. Zwar kann im Rahmen dieses Beitrags keine umfassende Rekonstruktion der einzelnen Fälle in ihrer Komplexität vorgenommen werden, doch soll ihre ausschnitthafte Analyse exemplarisch skizzieren, wie vegane Moralisierung hier praktiziert und plausibilisiert wird. Es soll dargestellt werden, dass mit der Überwindung des Tierkonsums zwar Universalisierungsansprüche einhergehen (können), dabei aber explizite Abgrenzungen von (militanten oder radikalen) Missionierungsbestrebungen rekonstruiert werden können. Diese Abgrenzungsprozesse im Veganismus verweisen darauf, dass militante Praxen, dogmatische Weltbilder oder totalitäre Selbstkonstruktionen, die eine geschlossene Struktur einer kommunikativen Welt- und Selbstbeziehung (Straub 2016) aufweisen, von den Akteur*innen selbst als dem Feld inhärent aufgefasst werden. 


\subsection{Transformationsansprüche und die Plausibilisierung von Moralisierung- Die Überwindung des Tierkonsums}

Zunächst wird skizziert, wie vegane Moralisierungspraktiken im Kontext der ausgewählten Erzählungen plausibilisiert und legitimiert werden. Hier ist anzumerken, dass Überzeugungen von der Handlungspraxis mit konstituiert und diese miteinander verwobenen Ebenen der Plausibilisierung, Legitimierung und konkreten Ausgestaltung von Moralisierungspraktiken im Veganismus nur zu analytischen Zwecken nacheinander betrachtet werden. Neben der Moralisierungsfähigkeit spielt hier die angenommene Notwendigkeit eine wesentliche Rolle. Dabei geht es in allen Interviews, von denen hier zwei exemplarisch betrachtet werden, immer wieder um die Aufhebung eines als defizitär gedeuteten gesellschaftlichen Zustandes des Tierkonsums, der sich (unhinterfragt) in den Deutungsmustern und Lebenspraxen einer Mehrheit der gesellschaftlichen Mitglieder abzeichne. Ein tiefes Misstrauen gegenüber einer wahrgenommenen Intransparenz bestimmter Strukturen und Prozesse in der Gesellschaft wird artikuliert sowie eine damit verbundene Unwissenheit der Menschen konstatiert und kritisiert - und (eigene) vegane Überzeugungen und Praxen als Ausweg formuliert. In diesem Zusammenhang kann mitunter an die Überlegungen von Joy (2017) angeknüpft werden, die soziale (Ver-)Lernprozesse für eine fehlende Wahrnehmung von Leid und Ungerechtigkeit bei sogenannten Nutztieren herausstellt. Solidarisierung und Übernahme von Verantwortung werden der eigenen individuellen veganen Lebenspraxis zugeschrieben, die durch den Verzicht auf Tierkonsum als progressiv und emanzipatorisch gedeutet wird. So heißt es im Interview mit Ben:

»Das sind alles so Dinge, wo wir schlichtweg verarscht werden auf Deutsch gesagt. Wo wir, wo wir durch die Industrie, äh einfach belogen werden nach Strich und Faden. Äh das ist Teil unserer Kultur, Teil unserer äh, Teil unserer Erziehung und man müsste einfach mal nur, auch da wieder Deep-Looking, mal sein Hirn einschalten, seinen gesunden Menschenverstand, sich wirklich mal als Krone der Schöpfung betätigen und sagen: Ich hab wirklich das bestentwickelteste Gehirn, setze ich es doch mal ein und überlege, ob das, was ich tue, gut ist und richtig ist. Für mich und für die Lebewesen um mich herum. Der Albert Einstein hat mal nen schönen, hat mal nen Brief geschrieben. Ich glaub das müsste in den 50er Jahren gewesen sein, ich weiß nicht mehr so genau und hat gesagt: Der Mensch, äh, das geht auch so ein bisschen in Speziesismus, was wir eben hatten, der Mensch betrachtet sich als losgelöst vom Rest, als etwas außerhalb des Restes und so ungefähr gings in die Richtung. Ähm, krieg das Zitat jetzt nicht mehr so hundertprozentig zusammen, aber letztendlich gings darum, dass er gesagt hat, diese Sichtweise ist wie ein Gefängnis, ist wie die Illusion, ne. Wir schaffen uns damit letztendlich, wir grenzen uns ab und schaffen uns letztendlich ein Gefängnis. Und wir müssen unser Mitgefühl erweitern auf alle Lebe- 
wesen, um uns aus diesem Gefängnis zu befreien. Das hat Albert Einstein gesagt. Nicht irgend son esoterischer Spinner $\ll(B e n$ 2019, Z. 819-36).

Es zeigt sich zunächst, dass vegane Überzeugungen auf spezifische Weise mit anderen Orientierungen verknüpft werden können (»Deep-Looking« [ebd., Z. 822] als Begrifflichkeit aus dem Buddhismus) und dabei ein hohes Modifikationspotenzial aufweisen, welches im ersten Abschnitt als Mehrdeutigkeiten und mögliche Differenzen im Veganismus herausgestellt wurde. Hier spielen beispielsweise auch biografische Relevanzen eine Rolle. Eine Zuwendung zum Buddhismus wird in einer früheren Passage des Gesprächs vom Interviewpartner auf den eigenen Handlungsdruck in der Vergangenheit zurückgeführt, der aus unerfüllten Moralvorstellungen eines gerechten und friedvollen Mensch-Tier-Verhältnisses resultiere. Durch einen buddhistischen Leitfaden könne er den eigenen Handlungsdruck kanalisieren und motivationale Kraft generieren, wobei die Gefahr emotionaler Belastung immer mitschwinge. Dies wird im späteren Verlauf des Beitrags noch durch eine semantische Verschränkung der Metaphern Weggehen und Schalter umlegen verdeutlicht. Interessant ist, dass in dieser Interviewsequenz sowohl vernunft- als auch mitgefühlorientiert argumentiert wird. Der »gesunde Menschenverstand (ebd., Z. 806) wird als Werkzeug verstanden, zu urteilen und richtig zu handeln. Hier zeichnet sich eine anthropozentrische Sichtweise ab, es als Mensch besser zu wissen und Verantwortung für die Mitwelt übernehmen zu sollen. Zugleich ist diese - an Vorstellungen von Gesundheit geknüpfte - Vernunftbegabung eine rationalisierende Konstruktion, die wenig Raum für Kritik lässt, denn abweichende Denkmuster und Handlungspraxen ließen sich in Relation als krankhaft und unvernünftig kategorisieren. In der Konstruktion von Vernunft sind Machtverhältnisse eingeschrieben, die Überlegenheit und Unterlegenheit markieren. Falsches Handeln wird kontrastierend dazu der Industrie, Kultur und Erziehung zugeordnet, welches der Mensch reflektieren könne, indem er Kritik übt und sich einsetzt, um diese defizitären Zustände zu überwinden. Während abstrakte und anonyme Gesellschaftsstrukturen eine gewisse Ohnmacht generieren können, liegt in der Regulation intimer, sozialer Beziehungen das Potenzial für die Wahrnehmung eigener Handlungsfähigkeit und die Wirkungsweise eigener Handlungsmacht. Dieser Aspekt wird zu einem späteren Zeitpunkt vertieft. Inwiefern der Mensch »verarscht« (ebd., Z. 819) wird, bleibt zunächst unbestimmt, eine industrielle und kulturelle Verblendung wird nicht weiter expliziert, ergibt sich allerdings aus dem Gesamtkontext des Interviews und wird auch in dieser Sequenz an den Begriff des $\gg$ Speziesismus $\ll^{2}$ (ebd., Z. 828) rückgebunden - welchen der Interviewpartner im Vorfeld als Analogie zu Rassismus und Sexismus definiert, und als »Faustrecht« (ebd., Z. 316), als Recht des Stärkeren versteht, Lebewesen einer anderen Spezies zu diskriminieren, und auf dieser Grundlage kritisiert. Der Speziesismus ist dabei eine (seiner) Deutungsgrundlage(n), um den Fokuspunkt dieser Sequenz auszumachen: Mit Bezug 
auf Einstein artikuliert er eine Kritik an der Abspaltung des Menschen von seiner Mitwelt (die eine selektive und damit fälschliche Wahrnehmung der Wirklichkeit bedinge, und zu einer Begrenzung des Menschen führe), die nur durch die Erweiterung des Mitgefühls behoben werden könne (und müsse, wie er hier verdeutlicht). Hier zeichnen sich essenzialisierende Vorstellungen von Wahrheit und Wahrhaftigkeit ab, die an die Glaubwürdigkeit Einsteins als Wissenschaftler und eben nicht als »esoterischer Spinner« (ebd., Z. 836) geknüpft sind. Bei dem veganen Künstlerpaar spielt eine Kritik am Speziesismus ebenfalls eine Rolle und wird von Flo dabei explizit an die Logik des Arguments, an progressive und emanzipatorische Gedanken geknüpft, strukturelle Grenzen und Differenzen aufzuheben. Dabei verortet er retrospektiv seine erste nach außen gerichtete Positionierung gegen speziesistische Praktiken des Tierkonsums noch vor einer konkreten Umsetzung in neue Handlungsgewohnheiten:

»Dann haben wir uns also auch ähm, dann auch theoretisch mit diesem ganzen Background beschäftigt, sind dann auf so Begriffe wie Speziesismus gestoß- gestoßen und ich hab dann halt ähm, zur Eröffnung der Ausstellung ne Rede gehalten. Und hab dann gemeint irgendwie, dass ähm, Speziesismus demnächst genauso verpönt sein wird wie ähm Rassismus, oder ähm, Frauenfeindlichkeit und so. Also das es eigentlich so ne logische Weiterentwicklung ist von den Rechten. Also. Und das habe ich dann irgendwie, ja vor ner klatschenden Menge von 300/350 Leuten gesagt und kaum war das vorbei, kommt denn der eine vegane Künstler auf mich zu, der [Name des veganen Künstlers] und ähm, äh haut mir so auf die Schulter und meint so: $>$ Ja, jetzt hast du dich ja auch positioniert.< Und in dem Moment habe ich mir dann sofort auf die Zunge gebissen, weil mir dann klar war: Ja Fuck, jetzt kann ich hier aber nicht mehr beim Sommerfest irgendwie ne Bratwurst essen. Also dann war das- ging es bei mir jetzt um Glaubwürdigkeit« (Flo 2019, Z. 214-26).

Hier wird deutlich, dass die Reflexion von Selbstverständlichkeiten des Tierkonsums eine Anschlussfähigkeit zu Vorstellungen von Gleichheit und Gerechtigkeit hat, die als leere Signifikanten ${ }^{3}$ in gewissen Situationen und über manifeste (beispielsweise normierte) Differenzlinien hinaus moralisch aufgeladen werden können. Dabei spielt die Selbstkongruenz zwischen (artikulierter) Überzeugung und eigener Praxis (dazu auch Lantermann 2016) eine wesentliche Rolle, um Authentizität und moralische Integrität zu wahren - bedeutsam für das eigene Selbst und in Relationalität zu Anderen (und damit verwobenen Formen des Gewährens und Verwehrens von Anerkennung). Der Einbezug der Konsequenzen eigener Positionierung ist dabei affektiv gefärbt, die Imagination, keine Bratwurst mehr essen zu können, ist sowohl mit möglichen Einschränkungen eigenen Genuss- und Geschmackserlebens verbunden, verweist implizit aber auch auf die Scham, diese zu essen (und womöglich sogar dabei gesehen zu werden). Sowohl die »klatschende[n] Menge « (vgl. Flo 2019, Z. 220) als auch das anerkennende 
Schulterklopfen sind zudem Indikatoren für eine Selbstbestätigung, die in diesem Zusammenhang von dem Interviewpartner höher gewertet wird als der genussorientierte Konsum tierischer Produkte. Dieser kleine Ausschnitt der Rekonstruktion einer latenten Sinnebene im Rahmen der Narration zeigt auf, dass in der Polyvalenz (Boesch 2000, 2006) des Veganismus auch Bedeutungen existent sind, die den interviewten Personen nicht immer offen zugänglich sind. Erika Quabach (2017) hat sich den ideologischen und identitätsbildenden Aspekten des Veganismus aus psychoanalytischer Perspektive gewidmet und herausgearbeitet, dass neben rationalen, kognitiv bearbeiteten Motiven immer auch unbewusste Beweggründe eine Rolle spielen,

»die die Wahl für eine vegane Ernährungsform und die damit verwobene moralische Lebensweise attraktiv machen. Veganismus kann funktional sein, wenn es um die bewusste Bewältigung von psychischen Konflikten und Krisen geht. Er kann Bedeutungen besitzen, die sich entziehen, solange wir allein über moralische und politische Dimensionen veganer Überzeugungen und Orientierungen diskutieren « (ebd., 80).

Die Verschränkungen von moralischen Gedanken und Empfindungen, von Kognition und Emotion, von rationaler Argumentation und gefühlter Verbundenheit, von offen kommunizierten, aber zugleich auch subtil wirkenden Motiven lassen sich auch in den anderen Interviews herausarbeiten. Moralisch aufgeladene, negativ konnotierte Begrifflichkeiten wie Illusion und Gefängnis, oder in anderen Interviewsequenzen verwendete Metaphern der Krankheit, Sucht oder Störung, werden genutzt, um einen gegenwärtigen defizitären Zustand von Gesellschaft als Begrenzung des eigenen Selbst (Ausschaltung des Mitgefühls, fehlende Reflexion über die eigenen Handlungsfolgen) zu deuten und kontrastierend dazu einen moralischen Raum für vegane Denk- und Handlungsmuster zu öffnen. Der Begriff der »Sucht « beispielsweise dekonstruiert Vorstellungen von Geschmack, Genuss und Ästhetik (tierischer Produkte) und ist ebenso wie ein Begriff des Verzichts nur relational zu verstehen. Die Relationalität zum Tierkonsum konstituiert letztlich vegane Denk- und Handlungsmuster und Moralisierungsbestrebungen, den Tierkonsum zu überwinden. Die Kontingenz von Handlungen (Joas 1999; Straub 2016) verweist in diesem Zusammenhang darauf, dass sie auf gewisse Weise vorstrukturiert sind, aber zugleich auch strukturieren und dabei immer auf die Möglichkeit verweisen, auch anders zu handeln. In ihrer moralischen Konstitution sind sie dabei mit spezifischen Wissensbeständen verwoben, die einen Anspruch auf Wahrheit und Wahrhaftigkeit entfalten (können). Hier ist die Potenz(-ialität) von Handlungen verortet, die sowohl auf individueller als auch auf gesellschaftlicher Ebene (ganze Gesellschaften befinden sich in Momenten des Handelns [Joas 1999]) besteht und im Veganismus den eigenen Tierkonsum in der Vergangenheit als Referenz zur Selbsttransformation markiert (es ist anzumerken, dass alle Interviewpartner*innen in ihrer Vergangenheit 
tierische Produkte konsumiert haben). Im Sinne von Hans Joas (1999) entstehen Werte in Momenten der Selbstbindung und Selbsttranszendenz. Werte haben dabei immer eine affektive Bindewirkung und gehen über eine rationale Argumentation hinaus. In der ersten Interviewsequenz wird ein Wert des Mitgefühls weiter gefasst und das Selbst in Verbundenheit mit der (Tier-)Welt verortet, dabei besteht aber immer noch die Möglichkeit, neue Begrenzungen vorzunehmen (und beispielsweise bestimmte Menschen von diesem Wert des Mitgefühls auszuschließen). In der zweiten Sequenz ist die logische Einsicht einer Speziesismus-Kritik und einer damit verbundenen Kritik am Tierkonsum einer Umsetzung in Handlungsgewohnheiten noch vorgelagert. Miteinander verwobene (vegane) Denk- und Handlungsmuster dienen der Prüfung von richtigen und falschen sowie besseren und schlechteren Handlungen (vergangener, gegenwärtiger oder imaginierter zukünftiger eigener Praktiken, aber auch Praxen des Umfeldes oder der gesamten Gesellschaft) und haben dabei immer einen situativen und kreativen Moment inne. Im Veganismus sind absolute Gewissheiten unverfügbar, da sowohl vorreflexive, mitunter unbewusste, aber auch nicht-intendierte Handlungsfolgen hier eine Rolle spielen - dennoch können vegane Denkmuster als Wahrnehmungsfilter verstanden werden, normalisierte Vorstellungen eines Mensch-Tier-Natur-Verhältnisses in der Gesellschaft zu kritisieren, umzudeuten und in neue Gewohnheiten zu überführen. Der situative Moment veganer Denk- und Handlungsmuster wird von den Interviewpartner*innen insbesondere auf Ebene veganer Moralisierungsbestrebungen verortet (auch Joas [1999] spricht von einem Universalisierungspotenzial der Vorstellungen des [Ge-]Rechten, das im Kontext der Kommunikation auf Kooperationsproblematiken stößt und erst dann Revisionsbedarf transparent macht). Nachfolgend soll ein Aspekt von (veganer) Moralisierung vertieft werden: Als (strategisches) Kommunikationsmittel steht die Vermittlung eigener Überzeugungen im Vordergrund. Dabei spielt vor allem die Funktionalität eines externen Impulses (der für die Initiierung eigener Reflexion vergangener Selbstverständlichkeiten bedeutsam war) eine wesentliche Rolle und wird in die Vorstellung überführt, selbst eben dieser Impuls für den Anderen beziehungsweise die Andere zu sein. Hiermit sind auch Fragen eigener moralischer Integrität und Authentizität untrennbar verwoben.

\subsection{Die Situativität veganer Moralisierungspraktiken - Lernprozesse der Sensibilisierung und Verfeinerung}

Vegane Moralisierungsbestrebungen sind in den Interviews zwar von wesentlicher Relevanz, werden aber in expliziter Abgrenzung zu Formen der Radikalität, des Dogmatismus, der Militanz und Missionierung markiert. Dabei gehören (auch im Interviewkontext) abgewertete Moralisierungspraktiken - beispielsweise aggressive Rede, 
Provokation, Konfrontation mit Schockbildern aus der Nutztierhaltung ${ }^{4}$, moralische Anklage oder politische Instrumentalisierung - ebenso zum Feld wie Prozesse der Selbstverschließung (Lantermann 2016), die eine Verbreitung veganer Überzeugungen gar nicht mehr zwingend zum Ziel haben. In diesem Beitrag steht ein Anspruch der Veganisierung im Fokus, der sich insbesondere auf das soziale Umfeld bezieht und dabei Partner*innen, Familienangehörige, Freund*innen, Bekannte und Kolleg*innen zu potenziellen Adressat*innen macht. Eine retrospektive Kritik an eigenen militanten, aggressiven Moralisierungen lässt sich mehreren Interviews entnehmen. So heißt es bei Maja:

»Also, einmal war ich richtig aggressiv irgendwiiie, meiner Cousine gegenüber. Weil dann kommen natürlich dann so Argumente: >Ja, aber das Soja, das ähm, das - es wird ja im Regenwald, also - wird Regenwald abgeholzt. < Und, dann muss man eben immer sagen: $>$ Ja, aber das ist halt das Soja für die Tiere, das Tierfutter und so< und ähm, GENAU und dann hab ichs aber auch so GEMERKT, dass ich mich so aufge- aufgeheizt hab, irgendwie und das fand ich dann auch, hinterher tat mir das auch richtig leid so. Weils auch meine Cousine ist und die ist ja auch eigentlich ganz liiieb und so. Und, hab ich mich auch entschuldigt dann später, aber ich hab daraus eben auch gelernt, dass das überhaupt nicht notwendig ist, äh überhaupt sich so zu ereifern. Also, das ist immer ganz gut, auch, JA, wenn man gefragt wird, was zu sagen und zu argumentieren, aber, ich ich muss- also wie [Name des Partners] gerade schon gesagt hat: Ich muss ja nicht missionieren. Also die Leute, die, DIE GUCKEN GANZ GENAU, was man macht, was man isst, was man wie man lebt« (Maja 2019, Z. 529-41).

Hier wird deutlich, dass Moralisierungspraktiken durch Rekurs auf den biografischen Erfahrungshorizont im Verlauf der Zeit verfeinert werden. Mögliche Verletzungen des Gegenübers, die aus der eigenen Aggressivität resultieren, werden nachfolgend reflektiert und durch eine mitfühlende Perspektivenerweiterung einbezogen. Dieses mitunter strategische Vorgehen hat gleichzeitig eine dynamische Komponente, welche (die stellenweise nicht greifbaren) Gelingens- und Misslingensbedingungen innerer sowie äußerer Regulation beeinflusst. Wenn beispielsweise nicht die verniedlichte und sympathische Cousine zur Adressatin gemacht wird, stellt sich die Frage, ob Impulsivität nicht doch zu einem probaten Mittel werden könnte, um eigene Handlungsmacht offen zu demonstrieren. Quabach (2017) merkt an, dass der Veganismus als Lebenskonzept und Überzeugungssystem das sogenannte »Böse, das in der Machtausübung liegt « (ebd., 78) - also die Anteile an Aggressionen, Impulsen und anderen unbewussten Motiven -, niemals vollständig auflösen könne (eine Aggressionsverleugnung sei in diesem Zusammenhang daher nur eine projektive »Delegation des Bösen an andere « [ebd., 80]). So spielt auch im Kontext der wiederholt artikulierten Ablehnung von Mis- 
sionierungsbestrebungen ein Faktor eine wesentliche Rolle, der an Aspekte gefühlter Macht- und Ohnmacht andockt: Glauben heißt nicht Wissen. Die religiöse Semantik (die auch Lantermann [2016] verwendet, wenn er von Erweckungserlebnissen vegan lebender Menschen schreibt und religiös konnotierte Begrifflichkeiten wie [Selbst-]Offenbarung, Bekehrung oder Bekenntnis nutzt) verweist zwar darauf, dass es sich bei dem Veganismus um ein Glaubens- und Überzeugungssystem handelt, allerdings sind in diesem Zusammenhang - wie oben skizziert - auch rationale Argumentation, spezifische Wissensbestände und Wahrheitsansprüche von Relevanz (die mit Machtansprüchen verwoben sind). Im Vorfeld der zuvor zitierten Passage führt die Interviewpartnerin ihre eigene emotionale, aggressive Haltung bei Diskussionen zur Verteidigung ihrer veganen Überzeugung auf fehlendes Wissen zurück:

»Also mir fiels immer schwer, dann argumentativ, ähm immer dann das richtige zu saaagen, oder auf ne Frage etwas zu sagen, was dann auch hinhaut. Und, oft mit Zaaahlen bin ich auch nicht so fiiirm und für viele ist es ja ganz gut, wenn man natürlich dann sofort ne Zahl parat hat. Und das hatte ich oft nicht « (Maja 2019, Z. 522-26).

Die rationale Überführung gefühlter Gewissheiten in Zahlen, Daten und Fakten dient dem Abbau eigener Unsicherheiten und gewährleistet die Anschlussfähigkeit im Austausch von Argumenten. Darüber hinaus ist eine kognitive Bearbeitung (auch) an Prozesse der Selbstregulierung gekoppelt, eine kommunikative Öffnung zu gewährleisten und diese nicht durch eigene negative Affekte zu gefährden. Grenzen des rationalen Arguments werden durch Akte des Zeigens und des Vorlebens überwunden (»die gucken ganz genau was man macht, was man isst, wie man lebt « [ebd., Z. 541]) und eigene Überzeugungsarbeit situativ angepasst. Als eine wichtige Gelingensbedingung der Moralisierung ist Sensibilität für das Gegenüber vielleicht ebenso unverzichtbar wie für ein harmonisches, auf gegenseitige Anerkennung beruhendes menschliches Miteinander. Wie oben skizziert, kann diese Sensibilität aber auch strategisch-funktional konnotiert sein: unter anderem als zielorientiertes und machtvolles Bestreben, die Mitmenschen an eigene Sicht- und Lebensweisen anzugleichen und dabei erfahrungsbedingtes Wissen um Psychotechniken anzuwenden. Moralisierung wird im Zuge der retrospektiven Auseinandersetzung mit sozialen Konflikten im Zusammenhang mit der Verteidigung und Verbreitung eigener veganer Überzeugungen zu einer aus Lernprozessen erwachsenen Kompetenz, die wesentlich für das vegane Selbstverständnis ist. Dies zeigt sich auch im Interview mit Ben, der in diesem Zusammenhang eine ganz spezifische Semantik verwendet:

»Meine erste Ehe äh, da war ich vielleicht ein bisschen militanter noch, ich glaub meine damalige Frau fand das auch teilweise gar nicht lustig, wenn ich ihr dann immer Vorhal- 
tungen gemacht habe, wenn sie sich aus meiner Sicht nicht so ernährt hat, wie ich das für ethisch ok finde. Und als ich dann meine Frau [im Jahr XX] kennengelernt habe, ähm, meine jetzige Frau, war ich da etwas moderater und hab gesagt, also ich werde ihr nicht irgendwas aufzwingen, sondern ähm, werde sie ihren eigenen Weg gehen lassen. Das war wahrscheinlich auch das Erfolgsrezept, dass wir bis heute verheiratet sind « (Ben 2019, Z. 68-74).

Auch hier lassen sich negativ konnotierte Vorstellungen veganer Moralisierung herausarbeiten, die biografische Relevanz haben. Das eigene, als militant gedeutete Vorgehen in der Vergangenheit fand seinen Ausdruck in ethischen Vorhaltungen und wurde durch Ansprüche der Homogenisierung begründet, die zu Beziehungskonflikten mit der Ehepartnerin führten. In der zweiten Ehe wird das Zusammenleben mit Zugeständnissen von Freiheitsräume verbunden und das eigene moderate Verhalten (vgl. ebd., 72) betont, was durch seine Relationalität implizit auf radikale Formen verweist, die zum Zeitpunkt des Interviews auf der Ebene des menschlichen Miteinanders abgewertet werden. Moralisierungsbestrebungen werden allerdings auch hier nicht aufgehoben, sondern nur angepasst:

»Und irgendwann ist dann meine Frau auch zum Vegetarier umgestiegen, das war- sie sagt selber, das war so wohl ne Szene, dass wir irgendwann mal, ähm an so ner Wiese vorbeigefahren sind, wo es Lämmer oder sowas gab, ne. Ähm und sie so, natürlich weil sie wirklich sehr sehr tierlieb ist, sagte: >Ach, guck doch mal die die Lämmer und wie schön<, und dann hab ich halt zu ihr gesagt: > Ja, aber du isst die. $<$ Und das war wohl bei ihr dann der der Schalter, der umgelegt wurde. Ich sag ja, jeder braucht irgendeinen, der den Schalter umlegt, ne. Bei ihr war es halt keiner in der Kindheit in der- ja in ihrem bisherigen Leben und ich war vielleicht dann derjenige, der bei ihr diesen Schalter umgelegt hat « (ebd., Z. 102-10).

Die verwendete Metaphorik Schalter umlegen (vgl. ebd., Z. 108ff.) kann neben Weg gehen (ebd., Z. 73 und 451) in Anlehnung an die Grounded Theory als In-Vivo-Code markiert werden (vgl. Codierprozeduren nach Strauss 1991 und Strauss und Corbin 1996; zit. nach Mey und Mruck 2009). Eine pragma-semantische Rekonstruktion (Straub 2010) fördert das Verständnis der Komplexität des Forschungsphänomens: So verweisen die ausgewählten Sprachbilder auf einen moralisch aufgeladenen Raum. Hier sind Vorstellungen notwendiger Transformationsprozesse verortet, welche die eigene Selbstveränderung gleichzeitig an Wahrhaftigkeit und Authentizität rückbinden (und von jeglichem Zwang loslösen). Gleichzeitig zeichnet sich hier allerdings auch ein Verständnis menschlichen Wahrnehmens und Erlebens ab, welche durch (sowohl nach innen als auch nach außen gerichtete) Willensbildung maßgeblich strukturiert und 
gesteuert werden können. In diesem Zusammenhang geht es darum, Mitmenschen den eigenen Weg gehen zu lassen (und durch Rekurs auf Aspekte des Mitgefühls, der Introspektion und Einfühlung sowohl das Leid der Tiere wahrzunehmen als auch empathisch für das soziale Umfeld zu sein) und ihnen gleichsam einen neuen Weg aufzuzeigen. Die Metaphorik des Schalter-Umlegens verweist dabei nicht nur auf einen intervenierenden Gestus, hier lässt sich auch ein spezifisches Machtverhältnis in der Beziehungskonstellation rekonstruieren: Die eigene Machtposition wird gestärkt, indem die Partnerin, der Metapher folgend, mechanisch bedient beziehungsweise kontrolliert wird (wobei von Ben anerkannt wird, dass auch sein Schalter im Vorfeld umgelegt wurde, was verdeutlicht, wie eng Selbst- und Fremdkontrolle miteinander verwoben sind). Idyllisch markierte Szenerien, wie die Wiese mit Lämmern, werden durch Einbezug der Konsequenzen des Handelns (Lämmer zu essen) als Illusion aufgedeckt, die durch diese Perspektivenerweiterung nicht mehr aufrechterhalten werden kann. Moralisierung zeigt sich hier als eine scheinbar objektiv feststellende Intervention (»Ja, aber $d u$ isst die« [ebd., Z. 106]), die positive Affizierungen durch Verbindung mit den Handlungskonsequenzen eigenen Konsums tierischer Produkte ins Negative umkehrt. Diese Affizierungen verweisen auf einen weiteren wesentlichen Aspekt im Kontext der Lernprozesse im Umgang mit dem eigenen Anspruch der Verbreitung veganer Denk- und Handlungsmuster, die insbesondere an oben bereits angemerkte Grenzen des rationalen Arguments stoßen. Genuss und Geschmack (mitunter auch als habituelle Prägemerkmale zu verstehen, die durch Verinnerlichung und Verkörperlichung subtil wirken) am Verzehr von Produkten tierischen Ursprungs spielen (auch) jenseits kognitiver und reflexiver Einwände gegen den Tierkonsum eine Rolle. In Bezug auf Veganismus ist die Mehrdeutigkeit beziehungsweise Um- oder Neudeutung von Vorstellungen des Verzichts von Relevanz. Flo führt aus:

»Also wir veganisieren eigentlich hauptsächlich dadurch, dass wir ähm, unglaublich lecker kochen. Also wir hatten letztens auch n Freund aus Süddeutschland hier und [Name der Partnerin] hat hier, ähm Kartoffelgulasch gemacht, mit Soja. Und der hat das überhaupt nicht auf die Kette gekriegt, dass das genauso schmeckt wie sein, wie sein Schweinegulasch, ne? Ist er überhaupt nicht drauf klargekommen, also hat er tausendmal probiert und hat dann hundertmal gefragt, wie sie das gemacht hat und dann so, ne? Und, ja und ähm, und DAS ist es eigentlich, wirklich den Leuten auch zu zeigen: >Ey, ihr könnt vegan oder vegetarisch leben, ohne verzichten zu müssen<, ne? « (Flo 2019, Z. 665-73)

Impulssetzungen sollen auch anderen behilflich sein, den Tierkonsum zu überwinden (und als negativ zu konnotieren). Die positive Deutung veganer Praxen ist eng damit verwoben. Flo und Maja verstehen sich dabei als Vermittler*innen neuer (veganer) Ge- 
nuss- und Geschmackserlebnisse. (Positiv konnotierte) Anregungen wie das Aufzeigen eigener veganer (Genuss-)Praktiken werden weniger als Einschränkung oder als Zwang markiert, sondern vielmehr als Eröffnung von Möglichkeitsräumen, den Mitmenschen etwas Gutes tun zu wollen oder ihren (Geschmacks-)Erlebnishorizont zu erweitern. Dies steht sowohl kontrastiv zu Vorwürfen von Missionierungsabsichten (Aufzeigen statt Anklagen) als auch zu Verzichtsvorstellungen veganer Lebenspraxen und fördert die (prozessual und situativ beeinflussten) Gelingensbedingungen von Moralisierung. Ziel ist es, bei den Mitmenschen ein inneres Bedürfnis, eine intrinsische Motivation zu wecken, die perspektivisch vegane Überzeugungen und Praxen als selbst gewollt und gewählt erscheinen lässt. Die Hinwendung zu veganen Denk- und Handlungsmustern wird darüber hinaus in anderen Interviewsequenzen als Zugewinn an persönlicher Freiheit, Perspektivenerweiterung und Steigerung der Selbstzufriedenheit durch die gefühlte Verbundenheit mit anderen Menschen, den Tieren und der Natur vermittelt. So wird ebenfalls eine gesteigerte Handlungsfähigkeit und Selbstwirksamkeit durch eine vegane Lebensweise unterstrichen. Daran knüpft auch das Verständnis eigener Moralisierung an. Im Interview mit Ben heißt es:

»Und ich bin überzeugt, äh, dass dieser, dass es grundsätzlich so ist, dass man den Leuten den eigenen Weg gehen lassen muss, dass man den Leuten ähm, dass man die nicht in ne, in eine Ecke drängt, ähm mit dem Zeigefinger auf sie zeigt, sondern ihnen einfach vv-versucht, ein Vorbild zu sein. Hört sich jetzt bescheuert an, weil ich bin ja jetzt nichts Besseres, nur weil ich vegan mich ernähre, ne. Aber, dass man einfach sagt: Ich versuche authentisch zu sein, etwas vorzuleben und dann können die Leute aus eigener Überzeugung sagen: Oh, das finde ich cool, ne« (Ben 2019, Z. 450-56).

Diese Vorstellungen der eigenen Vorbild- und Vorzeigefunktion resultieren mitunter aus Lernprozessen gelingender sowie misslingender Moralisierung, die immer auch das Ringen um die Einnahme der Perspektive des Gegenübers und eine kommunikative Öffnung beinhaltet. Doch nicht alle Konflikte lassen sich im Zuge dieser Prozessorientierung lösen. Gewisse Ambivalenzen bleiben bestehen und zeigen sich beispielsweise in der Unvereinbarkeit, gleichzeitig sowohl die Perspektive sogenannter Nutztiere einzunehmen, als auch die Perspektive derer zu verstehen, die weiterhin tierische Produkte konsumieren (so wie man selbst in der Vergangenheit). So illustriert Ben seine inneren Konflikte wie folgt:

»Also aus der Sicht des Miteinanders der Menschen finde ich dieses >Lass jeden seinen eigenen Weg gehen, äh, damit er gesichtswahrend und ohne Druck< und nicht das Gefühl haben, ich hab ja nur- bin ja nur jetzt Vegetarier geworden, weil es mir jemand quasi mich dazu gezwungen hat, dass das nachhaltiger und richtiger ist. Wenn man es aus der Sicht der 
Tiere sieht, ist es völlig falsch. Weil ich finde, da muss radikal was verändert werden, da ist dieses, ähm, der Hagen Rether hat mal gesagt von wegen persönliche Freiheit, ne, ähm, ist doch meine persönliche Freiheit, dass ich Fleisch esse. Hat der was ziemlich Derbes, glaube ich gesagt, dass das also absolut nicht richtig ist, ne, weil wir reden hier von Tierleid, wir reden hier von Tieren, die massivst gequält werden, unter unsäglichen Zuständen gehalten und getötet werden und da hört die persönliche Freiheit auf. Aus, also aus Sicht der Tiere muss ich das, was ich eben gesagt hab, dieses jeder geht seinen eigenen Weg, komplett umstellen und sagen, es müsste eigentlich ein gesetzliches Verbot her, ähm, dass Massentierhaltung nicht mehr erlaubt ist und so weiter und so fort. Krankenkassen müssten es belohnen, wenn Menschen sich vegan ernähren und äh, ähm, kein Fleisch mehr essen, solche Dinge. Also da wäre ich für wesentlich mehr oder wesentlich radikalere Maßnahmen, aus der Sicht der Tiere. Das ist, äh, da ist schon irgendwo ein Dilemma, was ich jetzt auch nicht lösen kann « (ebd., Z. 469-85).

Dieser Ruf nach einem Verbot - der hier zwar von der eigenen konkreten Lebenswelt losgelöst und auf eine abstrahierte Ebene gebracht wird - verweist darauf, dass der Handlungsmacht eigener veganen Überzeugungsarbeit zur Ausbreitung veganer Denkund Lebensweisen Grenzen gesetzt sind. Die Transformation machtvoller gesellschaftlicher Strukturen und Prozesse wird in diesem Zusammenhang höher gewichtet als die Freiwilligkeit der Wahl einer veganen Existenz. Spannungen resultieren aus den Handlungsspielräumen der nicht-veganen Anderen, welche zur Herstellung möglichst erfolgreicher Gelingensbedingungen von Moralisierung allerdings (partiell, temporär und strategisch) anerkannt werden. Auf alltagsweltlicher Ebene eröffnen sich letztlich (wahrgenommene) Möglichkeiten, eine Zukunfts- und Mehrheitsfähigkeit des Veganismus durch das Vorleben realer veganer Optionen zu fördern und die eigene Handlungsmacht zu stärken. In der Antizipation einer veganen Zukunft verbleibt die Verbreitung veganer Denk- und Handlungsmuster dabei ebenso kontingent (Joas 1999) wie die Normalisierung des Tierkonsums.

\section{Fazit und Ausblick}

In der empirischen Analyse wurde gezeigt, dass Moralisierung im Rahmen veganer Überzeugungsarbeit (auch) als reflexive Kommunikationsstrategie verstanden werden kann, die sich in der Auseinandersetzung mit (mitunter stereotypen) Missionierungsunterstellungen sowie anderen negativen Zuschreibungen (beispielsweise Veganismus als Verzicht) etabliert. Der Rekurs auf den eigenen biografischen Erfahrungshorizont sensibilisiert für mögliche Konflikte und dient zugleich der Verfeinerung gelingender Veganisierung. Moralisierung wird durch eine Verschränkung von Wissen, rationaler 
Argumentation und Mitgefühl beziehungsweise Empathiefähigkeit legitimiert und bisweilen sogar als notwendig eingefordert. Als Praxis wird sie mitunter in ihrer Aufklärungs- sowie Vorzeigefunktion hervorgehoben. In der Analyse ist darauf verwiesen worden, dass diese Legitimierung machtbesetzte Markierungen von Überlegenheit und Unterlegenheit sowohl de- als auch rekonstruiert. Moralisierung im hier untersuchten Feld ist affektiv gefärbt und fordert als zumindest partiell strategische Form der Kommunikation ein gewisses Maß an Selbstregulation ein, nicht zuletzt um in der Interaktion offen und anpassungsfähig zu werden beziehungsweise zu bleiben und potenzielle Konflikte in sozialen Beziehungen zu reduzieren. Zudem werden positive wie negative Affizierungen bisweilen auch strategisch genutzt, um Veganisierungen jenseits des rationalen Arguments zu fördern. Dabei geht es sowohl um die Spiegelung von als nicht wünschenswert erachteten Handlungsweisen (des Tierkonsums) als auch um das Vorleben veganer Handlungspraxen als neue (positiv konnotierte) Optionen, um sich von vergangenen Selbstverständlichkeiten zu lösen. In weiterer Forschung wäre beispielsweise zu untersuchen, wie Moralisierung im Veganismus auf Ebene des (kollektiven) Aktivismus plausibilisiert und praktiziert wird. Hier wäre unter anderem empirisch im Detail zu beleuchten, inwiefern affektgeladene, emotionale Ausdrucksformen wie Schweigen und Schreien als kommunikative Strategie des Moralisierens eingesetzt werden.

\section{Anmerkungen}

1 Bei den Namen handelt es sich um Pseudonyme.

2 Der von Richard Ryder (1983) geprägte Begriff des "Speziesimus« wird wiederholt im Kontext von Tierrechts-/Tierbefreiungsbewegungen genutzt und als Kritik vorgebracht, um auf eine essenzialisierte Form der Unterdrückung als minderwertig angesehener Wesen (Kaplan 1993; Singer 1996; siehe auch Schwarz 2005) hinzuweisen. Speziesismus legitimiert hierarchische (Gewalt-)Verhältnisse aufgrund der Zugehörigkeit zu einer spezifischen biologischen Art (Kaplan 1993), ohne dass Mensch-Tier-Beziehungen tatsächlich von Natur aus durch diese hierarchischen Unterschiede charakterisiert wären.

3 Ulrike Gatzemeier (2017) begreift werthaltige Kategorien wie Freiheit oder Gleichheit als leere Signifikanten (ebd., 74ff.), die unterbestimmt und nicht eindeutig festgelegt sind - daher beständig neu ausgelegt und ausgehandelt werden müssen, um Handlungen konkret zu beeinflussen (vgl. auch Straub und Ruppel 2017). Im Veganismus werden Handlungsgrenzen durch den Verzicht auf Tierprodukte vorbestimmt. Dennoch sind auch diese durch ein gewisses Maß an Situativität gekennzeichnet, insbesondere aufgrund ihrer Existenz in einer Gesellschaft, die den Tierkonsum normalisiert und verstärkt Spannungen im Rahmen der Aushandlung hervorruft.

4 Die einschlägige Kraft von bildlichen Eindrücken aus der Massentierhaltung, die Transparenz gewährleisten und Handlungsfolgen (des eigenen Konsums tierischer Produkte) nachzuvollziehen und eine auf sie bezogene Verantwortlichkeit wahrzunehmen helfen sollen, ist ein bedeutendes Medium im Kontext veganer Moralisierung. Auch wenn diese Bilder speziell 
im veganen Aktivismus genutzt werden, existieren interne Kontroversen bzgl. etwaiger negativer Folgen durch emotionale Belastung (Aspekte der Traumatisierung, Überforderung etc.).

\section{Literatur}

Adorno, Theodor und Max Horkheimer. 1988. Dialektik der Aufklärung. Philosophische Fragmente. Frankfurt a. M.: Fischer.

Boesch, Ernst E. 2000. Das lauernde Chaos. Mythen und Fiktionen im Alltag. Bern: Huber.

Boesch, Ernst Eduard. 2006. »Die Realität als Metapher«. Psychologie und Gesellschaftskritik 30 (34): 9-37.

Gatzemeier, Ulrike. 2017. Konflikt, Nation, Narration. Entwürfe des politisierten Selbst. Eine diskursivund biografieanalytische Studie zum »Sich-Ereignen der Nation « in Serbien 1987-1989. Bochum: Westdeutscher Universitätsverlag.

Glaser, Barney und Anselm Strauss. 1998. Grounded Theory. Strategien qualitativer Forschung. Bern: Huber (Orig. 1967. The Discovery of Grounded Theory. Strategies for Qualitative Research. New York: de Gruyter).

Grube, Angela. 2009. Vegane Lebensstile. Diskutiert im Rahmen einer qualitativen/quantitativen Studie. Stuttgart: Ibidem.

Guither, Harold D. 1998. Animal Rights. History and Scope of a Radical Social Movement. Carbondale: Southern Illinois University Press.

Herzog, Walter. 2018. »Moral«. In Stichwörter zur Kulturpsychologie, hrsg. v. Carlos Kölbl und Anna Sieben, 293-98. Gießen: Psychosozial-Verlag.

Hitzler, Ronald und Arne Niederbacher. 2010. Leben in Szenen. Formen juventiler Vergemeinschaftung heute. Wiesbaden: VS Verlag für Sozialwissenschaften.

Joas, Hans. 1999. Die Entstehung der Werte. Frankfurt a. M.: Suhrkamp.

Joy, Melanie. 2013. Warum wir Hunde lieben, Schweine essen und Kühe anziehen. Karnismus - eine Einführung. Münster: Compassion Media.

Joy, Melanie. 2017. Beyond Beliefs. A Guide to Improving Relationships and Communication for Vegans, Vegetarians, and Meat Eaters. Petaluma: Roundtree Press.

Kaplan, Helmut F. 1993. Leichenschmaus. Ethische Gründe für eine vegetarische Ernährung. Reinbek bei Hamburg: Rowohlt Taschenbuch.

Kohlberg, Lawrence. 1996. Die Psychologie der Moralentwicklung. Frankfurt a. M.: Suhrkamp.

Lantermann, Ernst-Dieter. 2016. Die radikalisierte Gesellschaft. Von der Logik des Fanatismus. München: Blessing.

Leitzmann, Claus und Markus Keller. 2013. Vegetarische Ernährung. Stuttgart: UTB.

Mey, Günter und Katja Mruck. 2009. »Methodologie und Methodik der Grounded Theory«. In Forschungsmethoden der Psychologie. Zwischen naturwissenschaftlichem Experiment und sozialwissenschaftlicher Hermeneutik, Band 3, hrsg. v. Wilhelm Kempf und Marcus Kiefer, 100-52. Berlin: Regener.

Quabach, Erika. 2017. „Vegan. Ideologische und identitätsbildende Aspekte einer Ernährungsweise«. Psychosozial 40 (2): 5-31.

Rabinow, Paul und William M. Sullivan. 1979. Interpretive Social Science. A Reader. Berkeley: University of California Press.

Regan, Tom. 2000. The Case for Animal Rights. Berkeley: University of California Press.

Regan, Tom. 2008. »Wie man Rechte für Tiere begründet«. In Texte zur Tierethik, hrsg. v. Ursula Wolf, 33-39. Stuttgart: Reclam. 
Rinas, Bernd-Udo. 2012. Veganismus. Ein postmoderner Anarchismus bei Jugendlichen? Berlin: Archiv der Jugendkulturen.

Rucht, Dieter. 1994. Modernisierung und neue soziale Bewegungen. Frankfurt \& New York: Campus.

Rude, Matthias. 2013. Antispeziesismus. Die Befreiung von Mensch und Tier in der Tierrechtsbewegung und der Linken. Stuttgart: Schmetterling.

Ryder, Richard. 1983. Victims of Science. The Use of Animals in Research. London: National Anti-Vivisection Society Limited.

Schütze, Fritz. 1983. »Biographieforschung und narratives Interview«. Neue Praxis 13 (3): 283-93. Zugriff am 15.03.2020. http://nbn-resolving.de/urn:nbn:de:0168-ssoar-53147.

Schwarz, Thomas. 2005. „Veganismus und das Recht der Tiere. Historische und theoretische Grundlagen sowie ausgewählte Fallstudien mit Tierrechtlern bzw. Veganern aus musikorientierten Jugendszenen«. In Eine Einführung in Jugendkulturen: Veganismus und Tattoos, hrsg. v. Wilfried Breyvogel, 69-165. Wiesbaden: VS Verlag für Sozialwissenschaften.

Singer, Peter. 1996. Animal Liberation. Die Befreiung der Tiere. Reinbek bei Hamburg: Rowohlt Taschenbuch.

Straub, Jürgen. 1999. Handlung, Interpretation, Kritik. Grundzüge einer textwissenschaftlichen Handlungs- und Kulturpsychologie. Berlin: de Gruyter.

Straub, Jürgen. 2010. „Das Verstehen kultureller Unterschiede. Relationale Hermeneutik und komparative Analysen in der Kulturpsychologie«. In Interpretative Sozialforschung und Kulturanalyse, hrsg. v. Gabrielle Cappai, Shingo Shimada und Jürgen Straub, 39-101. Bielefeld: Transcript.

Straub, Jürgen. 2016. Religiöser Glaube und säkulare Lebensformen im Dialog. Personale Identität und Kontingenz in pluralistischen Gesellschaften. Gießen: Psychosozial-Verlag.

Straub, Jürgen und Paul Sebastian Ruppel. 2017. »Einführung in den Themenschwerpunkt: Vegan und kerosinfrei. Eine moralische Subjektivierungsform im 21. Jahrhundert«. Psychosozial 40 (2): 5-31.

Straub, Jürgen und Pradeep Chakkarath. 2019. »Kulturpsychologie im Gespräch. Geschichte und Gegenwart eines Forschungsprogramms - Der Kontext der Hans-Kilian-Vorlesungen«. In Kulturpsychologie in interdisziplinärer Perspektive, hrsg. v. Jürgen Straub, Pradeep Chakkarath und Gala Rebane, 11-55. Gießen: Psychosozial-Verlag.

Strauss, Anselm. 1991. Grundlagen qualitativer Sozialforschung. Datenanalyse und Theoriebildung in der empirischen soziologischen Forschung. München: Fink.

Strauss, Anselm und Juliet Corbin. 1996. Grounded Theory. Grundlagen qualitativer Sozialforschung. Weinheim: Beltz.

Thrun, Rebecca. 2015. Jugendliche Identitätsbildung im Veganismus. Eine empirische Untersuchung im Raum Dortmund. Unveröffentlichte Masterarbeit Ruhr-Universität Bochum.

Thrun, Rebecca. 2017. »Jugendliche Identitäten im Veganismus. Zugänge zu einem erweiterten Selbstkonzept?«. Psychosozial 40 (4): 115-35.

Wimbauer, Christine und Mona Motakef. 2017. „Das Paarinterview in der soziologischen Paarforschung. Method(olog)ische und forschungspraktische Überlegungen«. Forum Qualitative Sozialforschung / Forum: Qualitative Social Research 18 (2), Art. 4. http://dx.doi.org/10.17169/ fqs-18.2.2671.

Witzel, Andreas. 2000. »Das problemzentrierte Interview«. Forum Qualitative Sozialforschung / Forum: Qualitative Social Research 1 (1): Art. 22. http://dx.doi.org/10.17169/fqs-1.1.1132.

Wolf, Ulrike. 2008. Texte zur Tierethik. Stuttgart: Reclam.

Yates, Luke. 2015. »Rethinking Prefiguration. Alternatives, Micropolitics and Goals in Social Movements«. Social Movement Studies 14 (1): 1-21. 
Rebecca Thrun

\section{Die Autorin}

Rebecca Thrun, M.A Sozialwissenschaft, seit 2017 Arbeit an einer Dissertation mit dem Schwerpunkt einer kulturpsychologischen Untersuchung veganer Lebensweisen, seit 2018 wissenschaftliche Mitarbeiterin am Lehrstuhl für Sozialtheorie und Sozialpsychologie (Prof. Dr. Jürgen Straub) an der Fakultät für Sozialwissenschaft, Ruhr-Universität Bochum.

Kontakt: Rebecca ThrunRuhr-Universität Bochum, Fakultät für Sozialwissenschaft, Lehrstuhl für Sozialtheorie und Sozialpsychologie, Gebäude GD 1/219, Universitätsstr. 150, D-44801 Bochum; E-Mail: rebecca.thrun@rub.de 